\title{
BMJ Open Providing additional information about the benefits of statins in a leaflet for patients with coronary heart disease: a qualitative study of the impact on attitudes and beliefs
}

\author{
Rebecca Dickinson, ${ }^{1}$ David K Raynor, ${ }^{1}$ Peter Knapp, ${ }^{2}$ Jan MacDonald ${ }^{3}$
}

To cite: Dickinson $\mathrm{R}$, Raynor DK, Knapp P, et al. Providing additional information about the benefits of statins in a leaflet for patients with coronary heart disease: a qualitative study of the impact on attitudes and beliefs. BMJ Open 2016;6:e012000. doi:10.1136/bmjopen-2016012000

- Prepublication history and additional material is available. To view please visit the journal (http://dx.doi.org/ 10.1136/bmjopen-2016012000).

Received 22 March 2016 Revised 3 November 2016 Accepted 8 November 2016 CrossMark

\footnotetext{
${ }^{1}$ School of Healthcare, University of Leeds, Leeds, UK

${ }^{2}$ Department of Health Sciences and The Hull York Medical School, University of York, York, UK

${ }^{3}$ Medicines and Healthcare Products Regulatory Agency, London, UK
}

Correspondence to Dr Rebecca Dickinson; r.dickinson@leeds.ac.uk

\section{ABSTRACT}

Objective: To explore the impact of providing additional information about the potential benefits of simvastatin in a patient leaflet on attitudes and beliefs.

Design: Interview-based study using a generic qualitative approach and framework analysis.

Participants: 21 participants receiving a prescription for simvastatin were recruited from a general practitioner practice (from a total of 120). 8 participants were women; the age range was 55-92.

Intervention: Participants were provided with leaflets showing one of 3 types of additional benefit information: (1) textual statement, (2) number needed to treat (NNT) or (3) natural frequency. Semistructured interviews explored patient's attitudes and beliefs.

Results: A descriptive narrative of preferences for format suggested patients prefer textual as opposed to numerical benefit information. Significant barriers to the acceptance of numerical benefit information included difficulty in understanding the numbers. Patients overestimated the benefits of statins and expressed surprise at the numerical information.

Conclusions: Textual information was preferred but numerical information, in particular in the form of a natural frequency, may help patients make judgements about their medicines. NNTs were found to be very difficult to understand. This raises the prospect that some patients might reject medicines because of disappointment with the perceived low benefits of their medicines. The self-reported impact on behaviour appeared minimal with reports of intentions to 'do what the doctor tells me'. Further research is needed to explore the impact of such statements on people who are yet to be prescribed a statin.

\section{INTRODUCTION}

For patients to take their medicines safely and effectively, it is important that they receive good quality information about their treatments. Across the European Union, all

\section{Strengths and limitations of this study}

- This study recruited actual users of medicines in order to explore their opinions on the inclusion of benefit information about their own treatments. Previous studies have generally used hypothetical samples or hypothetical medicines and situations.

- The participants recruited to this study were broadly representative of the general practice population who take simvastatin.

- We found that patients overestimated the benefits of statins and expressed surprise at the numerical information. Without information about the benefits and harms of their medicines, patients are not in a position to make informed choices about them.

- This study did not measure directly the impact of providing benefit information, it only explored self-reported behaviours. The self-reported impact on behaviour appeared minimal.

licensed medicines are required to be provided with written medicines information in the form of a patient information leaflet (PIL). Frequently, the PIL is the only written information a patient will receive about their medicines. ${ }^{1}$ PILs provide information about medicines such as:

1. What $X$ is and what it is used for

2. What you need to know before you take $\mathrm{X}$

3. How to take X

4. Possible side effects

5. How to store $\mathrm{X}$

6. Contents of the pack and other information.

PILs are available on http://www. medicines.org.uk. ${ }^{2}{ }^{3}$ Information about side effects (adverse effects) is presented in the format of a qualitative descriptor for five bands of risk alongside a natural frequency (such as 'common-affects less than 1 in 10 
people') as recommended in an European Medicines Agency Guideline. ${ }^{34}$

A common criticism of PILs is that they are too negative and can focus too heavily on side effect information. ${ }^{5}$ In order to redress the balance, medicine regulators have recently suggested that leaflets should also include information about the benefits of medicines. ${ }^{6}$ There is no universally agreed definition of what 'benefit' information encompasses, but it tends to include information about how the medicine works and its effectiveness.

Recently in the UK, the National Institute for Health and Care Excellence (NICE) has published guidance which recommends a wider use of statins, a class of lipid-lowering medications used in the treatment of cardiovascular disease. ${ }^{78}$ Critics of this change suggest that the benefits for low-risk patients often do not outweigh the possible side effects of the medicine, ${ }^{9}{ }^{10}$ as well as its effect of 'medicalising' healthy people.

NICE recommends that doctors give clear information to patients on the benefits of their treatments, ${ }^{11}$ but this can be challenging to do well. This is because there are different statistical methods for communicating benefits, some of which can be more persuasive than others. ${ }^{12}$ Furthermore, it can be complex to communicate these data to patients, as a number of patients struggle with the numerical concepts used to communicate benefits. ${ }^{13-15}$

The mandated PIL is the only regulated written information that a patient receives with their medicines and is included in every medicine pack. Therefore, such leaflets might be part of the solution to providing better quality information about the benefits of medicines to patients. However, currently little is known about preference for format or the potential impact of providing benefit information in a PIL.

Some studies have shown that presenting benefit information has a positive effect on positive judgement and the intention to take a medicine. ${ }^{16} 17$ Others have found that patients are more influenced by information on the adverse effects of medicines, rather than the benefits. ${ }^{18}$ Also, it has been shown that there can be significant concerns associated with the provision of benefit information to patients. A study from our research group found that the provision of numerical benefit information provoked strong feelings of shock and anxiety when the numerical benefits of an antiplatelet medicine were presented to a representative sample of older people.

However, such research has not yet been undertaken with people who are asked to read the benefit information about a medicine they are actually taking.

This study aimed to evaluate the following:

- The impact of providing benefit information about simvastatin in a PIL on the attitudes and beliefs that the actual users of simvastatin hold about their treatments.

- Patients' preferences for the inclusion and type of format of benefit information in a PIL.
METHODS

Design

This is a qualitative study using semistructured, face-to-face interviews conducted using a topic guide (see online supplementary appendix 1). Interviews lasted $\sim 1$ hour and were conducted in the patient's own home by the researcher ( $\mathrm{RD}-\mathrm{PhD}$ candidate). A qualitative approach is the most appropriate method to address the main objective, which is to explore the attitudes and beliefs of patients who might receive this type of information.

\section{Participants and setting}

Participants were recruited from a general practitioner (GP) practice in Bradford, UK, via a database search and were invited by letter to participate in audiorecorded interviews.

A purposive sampling strategy was employed to recruit patients already prescribed simvastatin for prior myocardial infarction (MI) or established coronary heart disease (CHD) (such as angina, unstable angina, previous coronary artery bypass graft (CABG) or angioplasty). The sample was intended to have similar demographic characteristics to sample recruited to the Heart Protection Study (HPS),${ }^{19}$ as this study was used to generate the data for the benefit statements. Participants were excluded if they were care home residents, receiving palliative care, had a cognitive impairment or were non-English speaking.

\section{Materials}

During the interviews, patients were shown three PILs produced for the purpose of the study but based on PILs commonly provided with simvastatin. Participants had as much time as they needed to review the PILs. Simvastatin was chosen for the following reasons:

- It is a commonly prescribed medicine-providing a large sampling frame from which to recruit.

- Simvastatin itself does not alleviate symptoms. Consequently, information provided about the chance of benefit may have particular significance for patients.

- Our previous research has shown that medicines perceived as having quite a small chance of benefit can create upset among participants. ${ }^{5}$ We wished to explore different magnitudes of benefit.

- Although the individual benefits may still be perceived as small, the population benefits of statin prescribing are potentially considerable. Understanding how patients perceive individual benefits might have an impact on willingness to take a treatment and consequently on population benefits.

Data were taken from the HPS, the largest published trial of simvastatin and which was funded by the UK Medical Research Council and the British Heart Foundation charity. ${ }^{19}$ We had identified a number of potential sources of data, including NICE guidelinesnational evidence-based clinical guidelines in the UK. 
NICE guidelines on statins for the prevention of cardiovascular events ${ }^{20}$ are based on a meta-analysis of studies into a variety of statins. Hence this could not be used, as the data needed to be specific to simvastatin. We identified four studies specific to simvastatin in NICE guidelines and chose the HPS because it provides effectiveness data for a secondary prevention or high-risk population. With the aim that the statements were as relevant to the individual as possible (without the need for a risk calculator which is not feasible in a PIL). The primary end points for which benefit data were presented were the likelihood of having a heart attack or stroke (and the standard equation for calculating number needed to treat (NNT) was used).

The leaflets were designed to look like PILs typically available in the UK. Simvastatin was given the hypothetical name 'Rebastatin' and each leaflet was marked with a highlighted section that stated 'This leaflet is for research purposes only'. The hypothetical name and highlighting were to ensure the leaflet was not mistaken for an actual PIL.

Three leaflets containing the following benefit statements were presented to the participants.

Textual: Rebastatin can reduce the chance of you having a heart attack or stroke. It does this by lowering levels of cholesterol and triglycerides in your blood.

Numerical: number needed to treat: If 17 people take Rebastatin over the next 5 years, one of them will be prevented from having a heart attack or stroke.

Numerical: natural frequency: In 100 people like you who do not take this medicine, 28 will have a heart attack or stroke. But if they all take Rebastatin over the next 5 years, 22 will have a heart attack or stroke.

\section{DATA ANALYSIS}

Data were organised and analysed by Framework analysis, using the following processes.

\section{Familiarisation}

After the interviews, field notes were made and initial categories for coding considered. Emerging themes were considered and discussed with the research team.

\section{Identifying a thematic framework}

One transcript was used to chart emerging codes which were developed into initial coding categories. These coding categories were checked against samples of the transcripts by three members of the research team (RD, JM and PK). The thematic framework was applied to each interview (see online supplementary appendix 2 ).

\section{Indexing}

The thematic framework was then applied to each interview and relevant data coded according to the framework. A software package, NVIVO, was used to manage the data (QSR International Pty, NVivo qualitative data analysis software. Version 9, 2010).

\section{Charting}

The indexed data were then sorted into charts. Each chart presented a main theme; every patient was represented by a row and each column was designated a subtheme. This allowed for all pertinent quotes from patients on a particular subtheme to be charted in a visually accessible way so that the researcher could view a summary of the data, yet view the different themes emerging by case and/or category.

\section{Mapping and interpretation}

The final stage saw a process of mapping and interpretation which was undertaken by RD and JM during two full-day meetings. RD and JM undertook a 'post-it note' exercise where each category and subcategory in the charts were summarised and arranged in emerging themes. Each researcher took a category and organise the emerging themes into subthemes until a coherent set of subthemes had been developed for each category. Field notes and mind maps were developed to present the emergent themes and the most-important themes identified from the framework.

\section{Research ethics consideration}

Research ethics approval was obtained from NRES Committee Yorkshire and the Humber-Humber Bridge: $13 / \mathrm{YH} / 0180$.

All participants received a PIL with details of the research prior to providing written informed consent prior to participation.

\section{RESULTS}

Letters were sent to 120 patients, of whom, 21 responded positively ( $17.5 \%$ positive response rate) and were interviewed: 20 in their own homes and 1 at their GP practice.

Eight of the participants were women. The age range was 55-92 (median age 75). Thirteen participants were educated to school level, three were educated to age 18 and five had completed higher education. Participants were currently receiving multiple prescribed medicines (range 4-10, median=7).

A range of views were obtained. The data were charted and organised into four broad themes which present a descriptive narrative of the range of attitudes and beliefs expressed regarding the benefit information.

1. Preferences for each format of benefit information.

2. Barriers to the inclusion of benefit information in PILs.

3. The impact on users of simvastatin of providing benefit information.

4. Desirable attributes for future benefit information.

\section{Preferences for each format of benefit information Textual}

The dominant perspective was that the textual format was preferable. The textual format was viewed as 
'positive', 'easy to read' and 'helpful' as it provided additional rationale for taking the medicine. It was perceived as reassuring and met participants' preferences for information that reinforced their decision to take the medicine.

The most positive one to read is obviously [textual statement] because it's just telling you that it's gonna reduce your chance and you think that's good. I stand a good chance of not having a heart attack or stroke. (P01, M, 75)

A less common perspective was that the textual format was perceived as insufficient and sometimes considered to be too cautious. It was reported that participants felt they could not develop a deeper understanding about the proportion of participants who would benefit from the treatment, especially when they compared the textual statement to one of the numerical statements:

I like to see that upfront [textual statement] but I'd perhaps be looking for perhaps something more...quantified benefits to back that up. I have... having, you know, talked about it, I think one of the quantified statements would be helpful. (P09, M, 72)

\section{Number needed to treat}

This format appeared to cause a great deal of confusion. A common occurrence was for participants to misinterpret that 1 extra person would be saved but the other 16 would have a heart attack.

It's a bit like saying a lottery, we've got seventeen people, one might be lucky and sixteen won't be lucky...The ball will roll and it could be that you could be the lucky one or not...your chances are one in seventeen, it almost says it's luck...it sounds like sixteen people are not gonna survive but one will. (P19, M, 76)

The provision of the NNT also had the potential to create an emotional response. It appeared to undermine participant's confidence in their medicines, created anxiety and removed hope:

The one in seventeen, I don't think would give me a lot of confidence. (P11, M, 77)

Less commonly, the participants responded positively about the NNT perceiving the statement as easy to read:

It's just clearer...you don't have to think about it. (P20, F, 55)

This was in comparison to the natural frequency, which often took some time to be understood.

\section{Natural frequency}

Although concerns were expressed about the natural frequency statements, it was viewed as helpful for those who could invest time and effort into understanding it. However, consistently participants continued to struggle to understand the natural frequency, and a group of participants did not understand it at all.

The natural frequency format tended to be regarded as positive in comparison to the NNT, as the numbers were perceived as more favourable. However, when considered alone it did not appear to convey large benefits to the patient and this was frequently viewed as disappointing:

It's not terribly impressive is it? A reduction from 28 to 22 ? But I suppose as a percentage it's about, mm, it's not a bad percentage, about 25 percent about 25 ...yeah, 28 down to $22 \ldots$ well, will that worry people being told those figures? I think a lot of people might be worried. They might think "oh, I'm the unlucky 1 in 5", we'll call 22 one in 5 . (P17, M, 81)

The level of benefit perceived from the natural frequency created a negative perception of the statement. However, it was the stated benefits of the medicine that were found disappointing, rather than the numerical format. Indeed, it was difficult for participants to separate their disappointment with the data from that associated with the format of the statement.

Not a very big decrease is it in people? 28 from 22. It's like saying $28 \%$ of you will have a heart attack if you take this tablet 22 of you will, so only $6 \%$ won't...I honestly don't think it's a large enough percentage of people, six. I think it should tell you a lot more will [benefit]... I think it's a bit on the low side. (P11, M, 77)

One perspective presented was that the natural frequency statement was viewed as sounding threatening; this undermined confidence in medicines:

I think that's a bit frightening because you'll be looking out and thinking am I going to be one of those 28 or 22 all the time I think. (P14, F, 66)

\section{Barriers to the inclusion of benefit information in PILs}

During the course of the interviews, the participants who said they would not typically read their leaflets engaged with and valued the benefit information, but still stated that they would be unlikely to read the leaflet in a natural situation.

I don't really think [I would read the leaflet] to be honest because at the moment I'm taking it because I've been told I need it. (P16, M, 68)

For those who stated they did not or would not read the leaflet (and therefore the benefit information), the stated reason was a lack of perceived relevance to their individual situation. The numerical information was viewed as irrelevant because those participants wanted to know the odds of what would happen to them individually and not the wider population.

It was also seen as potentially irrelevant; it was pointed out by participants that by the time patients receive the 
leaflet they have already made a decision to obtain the medicine. Therefore, timing of provision is an issue:

You wouldn't see this leaflet unless you were taking the medicine would you? (P05, M, 75)

Denial of the potential consequences of their own health condition was expressed. A recurrent view was that participants do not appear to want to hear the statistical facts about their medicine, as this was viewed as unpalatable.

I don't want to know that, I want them to tell me Simvastatin is doing you good and it will help prevent heart attacks, I don't want to know that $\mathrm{X}$ number are still gonna have a heart attack. (P01, M, 75)

A major barrier to accessing the benefit information was the challenge in understanding the benefit information. There were occurrences where participants made no attempt to try and understand it. However, a significant proportion was able to understand the provided numerical benefit information.

\section{The impact of providing benefit information for users of simvastatin \\ Satisfaction}

The participants reported that numerical benefit data had the potential to have a negative impact, provoking anxiety, worry and doubt; it was apparent that the information has the potential to be perceived as upsetting. The cause of this upset was the poor odds associated with the treatment:

It doesn't seem as if it helps a lot of people that, not in that length of time. There are only six people... Does that sound like sense? (P21, F, 84)

The textual benefit information was viewed as helpful and useful to know. Numerical information provided better clarity about treatments, more information about how the medicine was going to benefit them and how it would do this

I think you do need some facts and figures, it's interesting to know that if I take them, people who take statins are less likely and the percentage is worth knowing. (P05, M, 75)

A more exceptional perspective was that the information gave participant's confidence about their medicines and was encouraging and optimistic. The main benefit stated was that it met some participants' expectations of healthcare professionals having a duty to inform about the benefits of treatments. It satisfied their sense of 'needing to know':

Not everybody will want quantified information or quantified benefits, but I think equally there'll be a proportion who will look at the leaflet and will be looking for quantified information so they can actually say in quantified terms what the benefits are likely to be, so I would say yes. (P09, M, 72)

\section{Knowledge and understanding}

For many, the provision of benefit information in a PIL had no apparent impact on their knowledge or understanding about their medicines. There were several reasons for this. The information, particularly the numerical information, was viewed as too complicated. Others did not read the leaflet at all.

There were examples of participants who developed a deeper insight into the benefits of their medicine when reading the benefit statements for the purpose of the research study:

Honestly it has [helped me understand].... It's brought it home to me a bit... I'll take them with more joy [laughs]. (P16, M, 68)

For one participant in particular it put the concerns she had about her condition and treatment into context, which surprised her, but was very positive:

My father died at 44 and his brother died at $40 \ldots$ so I've always thought the percentage was a lot higher from my own personal experience, I thought when I was diagnosed that was it, I wasn't going to make it... But that's more reassuring to me because, alright 22 will have a heart attack or stroke but 78 won't if you are good and take the medicine. (P08, F, 56)

When the information did have an impact, this was sometimes a mix of a positive and negative effect. A minority view was that the provision of this type of numerical information helped with the development of a deeper understanding about the benefits of their treatments and encouraged participants to weigh up the advantages and disadvantages of treatments in more detail, but it also created more emotional responses and was often perceived as negative by the reader.

Despite the negative emotional responses, there was a sense of satisfaction that the numerical benefit information was included too. Participants wanted knowledge about the medicines to be made available.

I know it does make some people anxious...but I still think it's a good idea to put it in. (P06, M, 88)

\section{Decision-making and medicine-taking}

Concerns about the benefit information occasionally undermined the confidence that participants had in their medicines, leading to apprehensions that its inclusion in written medicines information might result in people being 'put off' from taking their medicines.

For this group of participants, the benefit information was in direct conflict with their positive beliefs about their medicines and desire for information reinforcing medicine taking. The numerical benefit information 
provided in the leaflets did not support patients with this, causing some anxiety and unease:

It doesn't really give me a great deal of confidence that I'm going to be the one who's going to be prevented from having the heart attack or stroke. (P09, M, 72)

In this sample, it did not appear that presenting such benefit information at this stage would have a significant influence on actual decision-making and medicinetaking for the participants. The biggest reported influence in this respect was the recommendation of their GP:

If the doctor told me to stick me head in a gas oven, I would do. I tend to believe in 'em. I've had good results and I mostly go along with 'em... (P06, M, 88)

A prevailing view was that as the decision about whether to take simvastatin or not had been made previously, sometimes decades before, the numerical benefit information was not necessarily viewed as something that could impact on decision-making. Instead, the numerical benefit information was something that was desired in order to reinforce the decision to take the medicine.

I'm not as impressed with that [numerical format], that's not as reassuring ... it's just numbers and it's not as personal. Reading [textual statement] it's speaking to me personally and it's giving me a bit of reassurance. (P20, F, 55)

One dominant perspective was that the participants would carry on taking simvastatin and hope that they would be one of those to benefit. The benefit information did not appear to have a negative impact on their approach to a medicine they were already taking.

\section{Desirable attributes of benefit information}

The benefit information did not appear to affect the balance of the leaflet-one of the reasons for its inclusion. One perspective presented was that it only served to make the leaflet appear more negative. Another view was that the side-effect information was perceived as so negative, the provision of benefit information could never balance that. The benefit information that was provided about simvastatin did not have sufficient positive impact to appear attractive:

I think the problem with these sorts of things is that there is too much emphasis on the bad side-effects. I know we've got to know, I know there is a duty of care to tell us all the bad things that might happen but I want to be drawn into what is good... I want the benefits to scream out at me. (P20, F, 55)

The appeal of qualifying words such as 'drop' to emphasise reduction in risk or 'increase' to emphasise improvements provided by the treatment was noted.
Participants wanted the information to be positive and to stress the benefits of the medicine.

I would like it to be more positive, take Rebastatin and you will benefit in the following terms...your likelihood of having a heart attack will drop from twenty-eight in a hundred to twenty-two in a hundred, I'd rather any information was presented in a positive way. (P09, M, TN)

There was a view that this could be achieved by using a combined textual and numerical statement. Having this textual statement as a precursor to a numerical statement appeared to reduce the disappointment and reinforce the benefits of the medicines that participants desire to hear more about:

If you're going to say it reduces the chances then tell people exactly how many it will reduce it by. (P08, F, 56)

This would mimic to a degree the way that side effect information is currently expressed in PILs (with textual and numerical information).

\section{DISCUSSION}

The provision of information about the potential benefits of a statin in a patient leaflet provoked complex and often emotive responses in people taking the medicine. This study demonstrates the range of opinions on, and the preferences for, format for benefit information. The dominant view was that textual information was preferable. In particular, there was a desire for additional information about the rationale for treatment.

It was also apparent that a small subset of patients developed a deeper insight about their medicines after reading the numerical statements. Natural frequencies appeared to be better suited to giving participants an understanding of the likelihood of benefit, which is a finding supported by previous studies. ${ }^{21-23}$ However, the natural frequency format was still too difficult for many participants to engage with.

Although the NNT was described as short and easy to read, it was frequently misinterpreted. This misinterpretation generated a considerable emotional response. The findings suggest that NNTs are not easily understood by lay people who frequently misinterpret the benefits of their treatment mean that 1 in 17 (in this case) will be saved, while the remainder come to harm. This is a finding that has been noted in other studies. ${ }^{24-27}$

The participants frequently overestimated the benefits of their medicine and when presented with numerical information about simvastatin this provoked surprise and disappointment. This response had been noted in a similar study with medicine takers who were also shown such numerical information but, importantly, not for a medicine they were actually taking. In that study, patients expressed shock and nihilism in response to reading benefit statements. ${ }^{5}$ It was concerning in that study that the routine provision of such information 
might promote decision-making based on the affect heuristic, rather than a rational consideration of the information.

This extreme emotional response was less noteworthy in this study of actual users of medicines. It was apparent that the participants were often shocked or disappointed with the benefit information and with the uncertainty associated with the effectiveness of the treatment. Previous experience with taking the medicine facilitated the appraisal of illness and treatment in the context of participants' current health state and appeared to play some role in mitigating this unease. ${ }^{28} 29$

This suggests that the users of medicines may not reject their treatments despite their concerns about perceived low benefits and will weigh up the likelihood of benefit and risk of harm of their treatments before altering their behaviour. This is not to say that the information should not be given; the concepts of patient empowerment and engagement are based on transparent provision of all relevant information. If the outcome of a reasoned assessment of the information is that a medicine is not right for an individual, then that decision must be respected.

Participants also desired information which reassured them and encouraged them to take their medicines. The numerical data on simvastatin failed to achieve this and was perceived as disappointing. However, it was apparent that the study participants still desire information about the potential benefits of their medicines, that is factual information which (may) reassure them and respond to their desire to continue to take their medicines as prescribed.

In the current study, the influence of the GP was also a strong moderating factor. Patients reported faith and trust in their GPs and the dominant perspective was that participants would do what their doctor tells them. Further research is needed to explore the influence of the GP. Concerns about patients rejecting medicines because of an emotional, rather than reasoned, response to the benefit information did not appear to be founded. Furthermore, the benefit information did not undermine the confidence that people had in their doctor's advice in such a way that it might lead to a change in medicine-taking.

It is possible that, as these patients were in receipt of long-term prescriptions for simvastatin, they might be influenced by the status quo bias which is the 'tendency to maintain a previous decision either by actively taking the default or by doing nothing, ${ }^{30}{ }^{31}$ It is possible that benefit information might impact differently on those who are considering a new treatment, that is, for patients who have not yet made a decision about taking it.

We know that over $70 \%$ of people read at least some of the PIL when they are first prescribed a medicine, ${ }^{32}$ but that leaves a significant proportion, as reflected in the current findings, for whom the inclusion of benefit information could not have an impact, as they do not read the leaflet. This is a significant barrier in engaging people about the benefits of their treatments by using written PILs, although other sources of information are available to people, notably online.

\section{Strengths and limitations of the research}

This study recruited actual users of medicines in order to explore their opinions on the inclusion of benefit information about their own treatments. Previous studies have generally used hypothetical samples or hypothetical medicines and situations. ${ }^{16}$ 33-36

The participants recruited to this study were broadly representative of the general practice population who take simvastatin. However, the sample is small and this group who volunteered to take part in research might hold different views to those who declined; consequently, there may be a degree of selection bias within the sample.

This study did not measure directly the impact of providing benefit information, it only explored self-reported behaviours. It is possible that while the participants might have reported that they would 'do what the doctor tells them' and remain adherent to their medicines, the provision of benefit information might in practice change medicine-taking behaviour. More research examining this possibility is needed.

Another limitation of the study is the choice of data on the benefits of statins to a targeted sample. This study used the findings from the HPS study, however, it is acknowledged that other data may alter the magnitude of benefit. The producers of benefit information for patients need to ensure a transparent process for choosing and presenting benefit data.

\section{CONCLUSIONS}

In 2014, NICE published guidance recommending a wider use of statins in the UK, reducing the prognostic threshold for initiating treatment, ${ }^{20}$ which will impact particularly on their use in primary prevention of heart and vascular disease. Opponents suggest that the benefits for low-risk patients often do not outweigh the possible side effects of the drug and that the risk-benefit information for statins is inadequately communicated. ${ }^{37} 38$

There are shortcomings with the availability and interpretation of data on the benefits and harms of statins and so uncertainty about their effectiveness. This will impact on the ability of healthcare professionals to effectively communicate risks and harms to patients and can be a barrier to informed decision-making. However, this study suggests that currently in the absence of the provision of numerical information about benefits many patients overestimate the benefits of their statins. Without information about the benefits and harms of their medicines, patients are not in a position to make informed choices about them.

While there are barriers to the use of a PIL, it is a mandatory and regulated piece of information that should be provided to every patient who is prescribed a licensed medicine. The PIL seems a logical place for the inclusion 
of information about the potential benefits of medicines -after all that is where the numerical information about side effects and their likelihood is to be found. The main disadvantages are timing (the PIL is provided when the medicine is dispensed and not during consultation) and visibility (only two-thirds of patients read a PIL when the medicine is first prescribed; the proportion is much lower for subsequent prescriptions).

The findings of this study suggest that people value the addition of extra textual information about the rationale of treatments. The provision of numerical information is more problematic as many people do not understand or value this information. However, there is a perspective that if the providers of medicines information know the numerical benefits of medicines, then this should be provided in the leaflet; not to do so would be to withhold information from patients. There was an expectation from participants that extra textual information about the rationale for a treatment would be enhanced by the provision of numerical information about its likely effectiveness.

Acknowledgements The authors thank Dr Rani Khatib and Dr Duncan Petty for their review of the benefit statements. They also thank the participants of the study and their GP practice. This study would not have been possible without the invaluable support of Dr June Raine, Director of Vigilance and Risk Management of Medicines, MHRA.

Contributors All authors contributed to the conception and design of the research. RD carried out all recruitment and interview activities. RD, JM, DKR and PK contributed to development of the analytical framework. RD and JM analysed the data. RD drafted the manuscript. All authors contributed to interpretation of the analysis, writing and reviewing the manuscript.

Funding RD's work on this study was supported by a doctoral studentship funded jointly by the UK Medicines and Healthcare products Regulatory Agency and the School of Healthcare, University of Leeds, UK.

Competing interests None declared.

Ethics approval NRES Committee Yorkshire \& the Humber-Humber Bridge: $13 / \mathrm{YH} / 0180$

Provenance and peer review Not commissioned; externally peer reviewed.

Data sharing statement No additional data are available.

Open Access This is an Open Access article distributed in accordance with the Creative Commons Attribution Non Commercial (CC BY-NC 4.0) license, which permits others to distribute, remix, adapt, build upon this work noncommercially, and license their derivative works on different terms, provided the original work is properly cited and the use is non-commercial. See: http:// creativecommons.org/licenses/by-nc/4.0/

\section{REFERENCES}

1. Medicines and Healthcare Products Regulatory Agency. Best practice guidance on patient information leaflets. London: Medicines and Healthcare products Regulatory Agency, 2012.

2. Dickinson D, Raynor DKT. Ask the patients-they may want to know more than you think. BMJ 2003;327:861.

3. Co-ordination Group for Mutual Recognition and Decentralized Procedures-Human. CMDh annotated QRD template for MC/DC procedures (based on version 8 of the QRD template for $\mathrm{CP}$ ), 2011. http://www.hma.eu/uploa

4. Harris K, Dickinson R, Raynor D, et al. Changes in side effect risk communication in patient information leaflets over the past decade: results of a survey. Drug Saf 2015;38:1-11.

5. Hamrosi K, Dickinson R, Knapp P, et al. It's for your benefit: exploring patients' opinions about the inclusion of textual and numerical benefit information in medicine leaflets. Int $J$ Pharm Pract 2012;21:216-25.
6. Medicines and Healthcare Products Regulatory Agency. Always read the leaflet: getting the best information with every medicine. The Stationary Office, 2005.

7. National Clinical Guideline Centre. Lipid modification: cardiovascular risk assessment and the modification of blood lipids for the primary and secondary prevention of cardiovascular disease - clinical guideline methods, evidence and recommendations. National Institute for Health and Care Excellence, 2014.

8. National Institute for Health and Care Excellence. Cardiovascular risk assessment and lipid modification-NICE quality standard [QS100]. National Institute for Health and Care Excellence, 2015.

9. McCartney M. The patient paradox. UK: Pinter and Martin, 2012.

10. Abramson JD, Rosenberg HG, Jewell N, et al. Should people at low risk of cardiovascular disease take a statin? BMJ 2013;347:f6123.

11. National Institute for Health and Care Excellence. Medicines adherence: involving patients in decisions about prescribed medicines and supporting adherence. NICE guidelines [CG76], UK: NICE, 2009

12. Akl E, Oxman A, Herrin J, et al. Using alternative statistical formats for presenting risks and risk reductions. Cochrane Database Syst Rev 2011;(3):CD006776.

13. Peters E, Hibbard J, Slovic $P$, et al. Numeracy skill and the communication, comprehension, and use of risk-benefit information. Health Aff 2007;26:741-8.

14. Lipkus IM, Samsa G, Rimer BK. General performance on a numeracy scale among highly educated samples. Med Decis Making 2001;21:37-44.

15. Gardner PH, McMillan B, Raynor DK, et al. The effect of numeracy on the comprehension of information about medicines in users of a patient information website. Patient Educ Couns 2011:83:398-403.

16. Bersellini $E$, Berry $D$. The benefits of providing benefit information: examining the effectiveness of provision of simple benefit statements on people's judgements about a medicine. Psychol Health 2007;22:61-82.

17. Amery WK. Coming full circle in pharmacovigilance: communicating safety information to patients through patient package inserts. Pharmacoepidemiol Drug Saf 1999;8:121-9.

18. Fried TR, Tinetti ME, Towle V, et al. Effects of benefits and harms on older persons willingness to take medication for primary cardiovascular prevention. Arch Intern Med 2011;171:923-8.

19. Heart Protection Study Collaborative Group. MRC/BHF Heart Protection Study of cholesterol lowering with simvastatin in 20536 high-risk individuals: a randomised placebocontrolled trial. Lancet 2002;360:7-22.

20. National Institute for Health and Care Excellence. Lipid modification: cardiovascular risk assessment and the modification of blood lipids for the primary and secondary prevention of cardiovascular disease. London: National Institute for Health and Care Excellence, 2014.

21. Girotto V, Gonzalez M. Chances and frequencies in probabilistic reasoning: rejoinder to Hoffrage, Gigerenzer, Krauss, and Martignon. Cognition 2002;84:353-9.

22. Gigerenzer $\mathrm{G}$. Reckoning with risk: learning to live with uncertainty. London: Penguin Books, 2002.

23. Galesic M, Gigerenzer G, Straubinger N. Natural frequencies help older adults and people with low numeracy to evaluate medical screening tests. Med Decis Making 2002;29:368-71.

24. Christensen PM, Kristiansen IS. Number-needed-to-treat (NNT)needs treatment with care. Basic Clin Pharmacol Toxicol 2006;99:12-16.

25. Halvorsen PA, Selmer R, Kristiansen IS. Different ways to describe the benefits of risk-reducing treatments: a randomized trial. Ann Intern Med 2007;146:848-56.

26. Carling CL, Kristoffersen DT, Montori VM, et al. The effect of alternative summary statistics for communicating risk reduction on decisions about taking statins: a randomized trial. PLoS Med 2009:6:e1000134

27. Kristiansen IS, Gyrd-Hansen D, Nexøe J, et al. Number needed to treat: easily understood and intuitively meaningful?: theoretical considerations and a randomized trial. J Clin Epidemiol 2002;55:888-92.

28. Cameron LD, Jago L. Emotion regulation interventions: a common-sense model approach. Br J Health Psychol 2008;13:215-21.

29. Cameron LD, Leventhal $\mathrm{H}$. The self-regulation of health and illness behaviour. London: Routledge, 2003.

30. Suri G, Sheppes G, Schwartz C, et al. Patient inertia and the status quo bias: when an inferior option is preferred. Psychol Sci 2013:24:1763-9.

31. Samuelson W, Zeckhauser R. Status quo bias in decision making. J Risk Uncertainty 1988;1:7-59. 
32. Raynor DK, Silcock J, Knapp P, et al. How do patients use medicine information leaflets in the UK? Int J Pharm Pract 2007;15:209-18.

33. Zikmund-Fisher BJ, Fagerlin A, Ubel PA. What's time got to do with it? Inattention to duration in interpretation of survival graphs. Risk Anal 2005;25:589-95.

34. Gyrd-Hansen D, Kristiansen IS, Nexoe J, et al. How do individuals apply risk information when choosing among health care interventions? Risk Anal 2003;23:697-704.
35. Kristiansen IS, Gyrd-Hansen D, Nexoe J, et al. Number needed to treat: easily understood and intuitively meaningful? Theoretical considerations and a randomized trial. J Clin Epidemiol 2002;55:888-92.

36. Garcia-Retamero R, Galesic M. How to reduce the effect of framing on messages about health. J Gen Intern Med 2010;25:1323-9.

37. McCartney M. Statins for all? BMJ 2012;345:e6044.

38. Godlee F. Adverse effects of statins. BMJ 2014;348:g3306. 